\title{
Associations between Intra-Pancreatic Fat Deposition, Pancreas Size, and Pancreatic Enzymes in Health and after an Attack of Acute Pancreatitis
}

\author{
Juyeon Ko Loren Skudder-Hill Sunitha Priya Wandia Kimita \\ Sakina H. Bharmal Maxim S. Petrov \\ School of Medicine, University of Auckland, Auckland, New Zealand
}

\section{Keywords}

Intra-pancreatic fat deposition · Pancreas volume - Pancreas size $\cdot$ Pancreatic enzymes $\cdot$ Body composition

\begin{abstract}
Introduction: Ectopic fat deposition in the pancreas is involved in the pathogenesis of metabolic sequelae following an attack of pancreatitis. However, its relationship with the exocrine pancreas has never been explored in this setting. The aim was to investigate the associations between intrapancreatic fat deposition (IPFD), pancreas size, and pancreatic enzymes. Methods: This cross-sectional study recruited individuals with a history of acute pancreatitis and healthy controls. All participants underwent $3 \mathrm{~T}$ magnetic resonance imaging, from which IPFD, total pancreas volume (TPV), and pancreas diameters (across the head, body, and tail) were measured independently by 2 raters in a blinded fashion. Circulating levels of pancreatic amylase, pancreatic lipase, and chymotrypsin were measured in a fasted state. A series of linear regression analyses was conducted, accounting for possible confounders. Results: A total of 108 individuals with pancreatitis and 60 healthy controls were studied. There was a statistically significant difference in IPFD ( $p<$ $0.001)$, but not in TPV $(p=0.389)$, between the groups. In the
\end{abstract}

karger@karger.com www.karger.com/ofa

Karger $\stackrel{\text { ' }}{5}$

GOPEN ACCESS
(C) 2021 The Author(s)

Published by S. Karger AG, Basel

This is an Open Access article licensed under the Creative Commons Attribution-NonCommercial-4.0 International License (CC BY-NC) (http://www.karger.com/Services/OpenAccessLicense), applicable to the online version of the article only. Usage and distribution for commercial purposes requires written permission. post-pancreatitis group, IPFD was significantly inversely associated with pancreas tail diameter $(\beta=-0.736, p=0.036$ in the most adjusted model). In the control group, IPFD was significantly inversely associated with TPV $(\beta=-3.557, p=$ 0.026 in the most adjusted model). Levels of pancreatic amylase were significantly directly associated with pancreas tail diameter in the post-pancreatitis group $(\beta=3.891, p=0.042$ in the most adjusted model), whereas levels of pancreatic lipase were significantly inversely associated with TPV in the control group ( $\beta=-10.533, p=0.024$ in the most adjusted model). Conclusion: Increased IPFD in individuals after an attack of pancreatitis is associated with reduced pancreas tail diameter, which is in turn associated with reduced circulating levels of pancreatic amylase. The relationship between IPFD and the exocrine pancreas warrants further investigations.

(c) 2021 The Author(s)

Published by S. Karger AG, Basel

\section{Introduction}

Acute pancreatitis (AP) is a complex disease with a variable course that is often difficult to predict, with an incidence of 34 people per 100,000 in the general population worldwide $[1,2]$. Gallstones and alcohol abuse are 
the most common causes of AP [2]. While the old belief was that AP is a self-limiting disease, it is now appreciated that individuals after AP often develop metabolic sequelae [2]. For example, a 2020 prospective longitudinal cohort study with regular follow-ups (as part of the LACERTA project by the Clinical and epidemiOlogical inveStigations in Metabolism, nutritiOn, and pancreatic diseaseS [COSMOS] group) demonstrated that $43 \%$ of nondiabetic patients with AP developed new-onset prediabetes or diabetes within 2 years of hospital discharge [3]. This is in accord with evidence from a 2014 meta-analysis of cross-sectional and case-control studies by the COSMOS group that found that up to $40 \%$ of AP patients without a prior history of diabetes developed prediabetes or diabetes after the first attack of AP [4]. Further, a 2021 population-based study (as part of the NORMA project by the COSMOS group) showed that exocrine pancreatic dysfunction is common after an attack of pancreatitis, and it represents a significant risk factor for new-onset diabetes in this setting [5]. Although the pathogenesis of endocrine and exocrine pancreatic dysfunction in individuals after AP is complex and its predisposing factors are yet to be fully elucidated, recent studies suggested that one of its key drivers is changes in pancreas morphology - specifically, increased intra-pancreatic fat deposition (IPFD) and reduced total pancreas volume (TPV) [6-8].

Morphological changes in the pancreas are increasingly recognized as a quantifiable comprehensive measure of pancreas health, thanks to advances in imaging techniques such as magnetic resonance (MR) imaging [9-12]. A 2017 meta-analysis of 24 human studies by the COSMOS group revealed that increased IPFD was significantly associated with several metabolic disorders [13]. Moreover, a 2020 meta-analysis of 20 human studies by the COSMOS group showed that low circulating levels of pancreatic enzymes characterize many metabolic disorders, including fatty pancreas disease [14]. Similarly, changes in pancreas size (as determined by TPV, pancreas diameters, areas, and lengths) were reported in a broad range of disorders [15]. A 2018 systematic review of 27 human studies by the COSMOS group demonstrated significant changes in TPV in people with diabetes. Specifically, compared with healthy individuals, large (the effect size of 1.3) and medium (the effect size of 0.4) reductions of TPV were shown in individuals with type 1 diabetes and type 2 diabetes, respectively [10]. Also, a 2019 MR study by the COSMOS group reported that both TPV and pancreas tail diameter (PTD) reductions were associated with increased recurrence of AP [8]. To the best of our knowledge, no study to date has explored the inter-relationships between pancreas size and IPFD in the post-pancreatitis setting. The present study aimed to investigate the associations between IPFD, pancreas size, and pancreatic enzymes in individuals after an attack of $\mathrm{AP}$ and healthy individuals.

\section{Methods}

\section{Study Population}

This was a cross-sectional study nested into prospective longitudinal cohort study as part of the ARIES project, approved by the Health and Disability Ethics Committee (13/STH/182) [8, 16-18]. Adult individuals residing in Auckland (New Zealand) were eligible for the study if they had a primary diagnosis of AP established prospectively based on the international guidelines [19]. Individuals were excluded if they had an attack of AP within 3 months prior to enrolment into the study, post-endoscopic retrograde cholangiopancreatography pancreatitis, chronic pancreatitis, hereditary pancreatitis, autoimmune pancreatitis, pancreatic cyst, congenital anomalies of the pancreas, cystic fibrosis, pancreatic lipomatosis or lipomatous pseudohypertrophy, malignancy, cognitive impairment, received surgical, endoscopic, or radiological interventions involving the pancreas, received steroid therapy, had metallic foreign body implants, heart pacemakers, or other implantable electronic devices, or were pregnant.

Healthy controls were also recruited. They had no personal history or symptoms of diseases of the exocrine pancreas or diabetes, no upper abdominal pain or nausea, no history of acute infectious or inflammatory conditions requiring medical treatment or evaluation in the preceding 6 months, no family history of diseases of the exocrine pancreas, diabetes, coeliac disease, or cystic fibrosis. All participants provided informed consent.

\section{Acquisition of MR Imaging}

All participants underwent abdominal MR imaging at the Centre for Advanced Magnetic Resonance Imaging (University of Auckland, Auckland, New Zealand). A 3.0 Tesla MAGNETOM Skyra scanner (Siemens, Erlangen, Germany) was used to acquire MR images in all study participants. Participants underwent MR imaging wholly and exclusively for the purpose of the ARIES project [18]. Axial T1-weighted volumetric interpolated breath-hold examination Dixon sequence was used with the following parameters: true form abdomen shim mode; FOV , $420 \mathrm{~mm}$; flip angle, $9^{\circ}$; slice thickness, 3 and $5 \mathrm{~mm}$. Four types of images were generated: in-phase, out-of-phase, fat-only, and water-only images. These images were retrieved from the MR scanner and exported as DICOM files for analyses using ImageJ software (National Institutes of Health, Bethesda, MD, USA).

The studied MR imaging-derived variables included IPFD, $\mathrm{TPV}$, and pancreas diameters. Two independent raters performed all the measurements blinded to the study group allocation, and the average value of 2 measurements was used for the statistical analyses. Intra-class correlation coefficients (ICCs) were calculated to assess the inter-rater reliability. The inter-rater reliability was deemed excellent if the ICC was more than 0.90 [8]. 


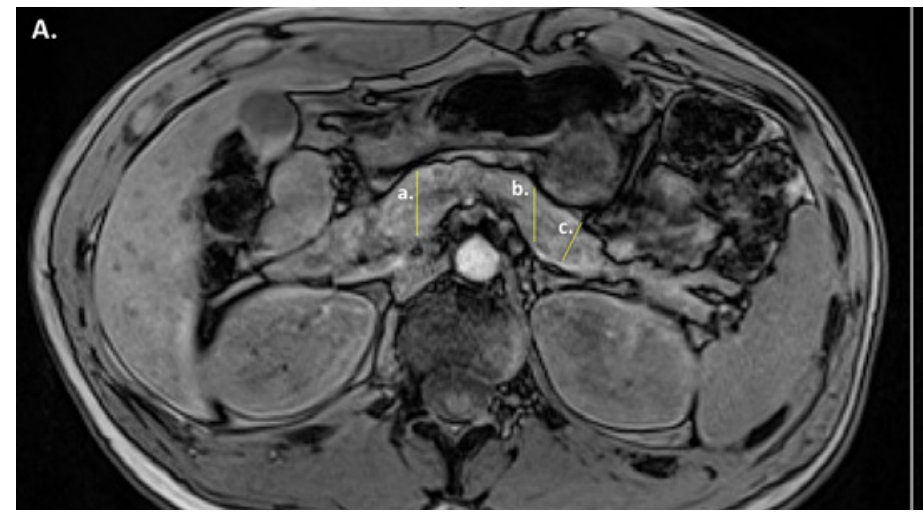

Fig. 1. Measurements of pancreas diameters $(\mathbf{A})$ and intra-pancreatic fat deposition (B) in a 30-year-old healthy woman with a body mass index of $20.0 \mathrm{~kg} / \mathrm{m}^{2}$. Out-of-phase images are presented. Regions of interest were placed in the head, body, and tail of the pancreas. The anteroposterior diameter of the pancreas head was measured in line with the right-most point of the confluence of the

\section{Measurement of Intra-Pancreatic Fat Deposition}

IPFD was measured based on fat-only images, as described in detail elsewhere $[7,18]$. Briefly, 2 candidate slices from a series of 5 -mm-thick slices with clear visualization of the pancreas were chosen from a series of abdominal scans. Out-of-phase images were used for confirmation of region of interest placement. Three regions of interest $\left(100 \mathrm{~mm}^{2}\right)$ were placed in the head, body, and tail regions of the pancreas to determine IPFD (Fig. 1). A thresholding range of $1-20 \%$ was applied to prevent the potential inclusion of non-parenchymal tissues (such as visceral fat, the main pancreatic duct, and blood vessels) within the selected regions of interest [20]. IPFD (\%) was calculated by taking the average of measurements of the 2 slices.

\section{Measurement of Total Pancreas Volume}

Quantification of TPV was based on out-of-phase images of 5 $\mathrm{mm}$ slice thickness, as described in detail elsewhere [8]. Briefly, the pancreas outline was traced on each slice in which it was visible, and the associated pixel content was calculated. Water-only images served as the reference for each slice, and care was taken to exclude surrounding blood vessels. TPV, measured in cubic centimetres $\left(\mathrm{cm}^{3}\right)$, was obtained by multiplying the pixel content from all the slices in series with the pixel area and slice thickness.

\section{Measurement of Pancreas Diameters}

Pancreas diameters were measured in millimetres ( $\mathrm{mm}$ ) using out-of-phase images with 3 -mm slice thickness (Fig. 1). The pancreas head diameter (PHD) was measured in line with the right-most point of the confluence of the superior mesenteric and splenic veins [8]. The pancreas body diameter (PBD) was measured in line with the left lateral border of the lumbar vertebrae [8]. The PTD was measured as a line perpendicular to the organ midline, at a point $20 \mathrm{~mm}$ from the distal-most point of the pancreas in the slice. The slice used for PTD measurement was the one that offered the best tail visualization (within close proximity to the splenic hilum). Care was taken to exclude surrounding blood vessels.

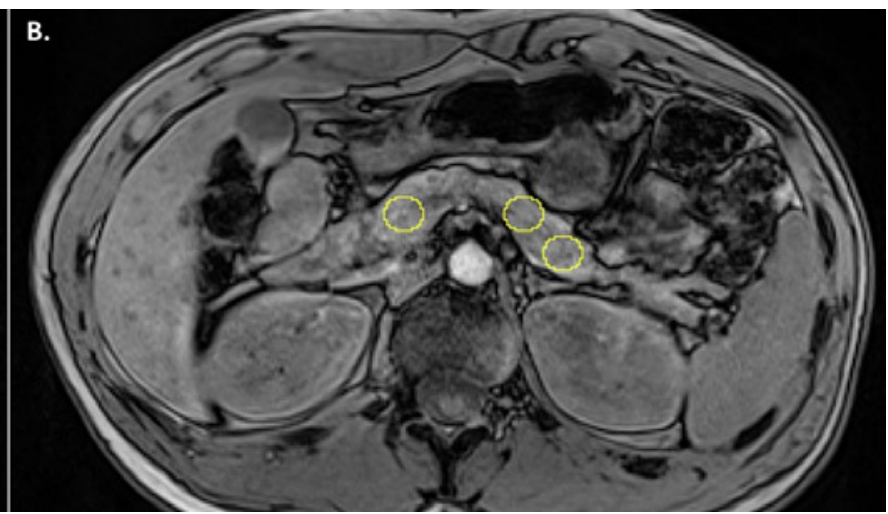

superior mesenteric and splenic veins (a); the anteroposterior diameter of the pancreas body was measured in line with the left lateral border of the lumbar vertebrae (b); the tail diameter of the pancreas was measured as a line perpendicular to the organ midline, at a point $20 \mathrm{~mm}$ from the distal-most point of the pancreas in the slice (c).

\section{Measurement of Pancreatic Enzymes}

Venous blood samples were obtained from each participant (after at least $8 \mathrm{~h}$ of fasting) at the time of their participation in the ARIES project. These blood samples were centrifuged $4,000 \mathrm{~g}$ for $7.5 \mathrm{~min}$ at $4^{\circ} \mathrm{C}$; the plasma was separated into aliquots and stored at $-80^{\circ} \mathrm{C}$ until further use. Pancreatic amylase was measured in the plasma using the Reflotron ${ }^{\circledR}$ Plus reflectance photometer (Roche ${ }^{\circledR}$, Basel, Switzerland), and results were expressed in U/L. Pancreatic lipase and chymotrypsin were measured using sandwich ELISA. Pancreatic lipase was measured using the Cloud-Clone Corporation ELISA kit (Houston, TX, USA), and results were expressed in $\mathrm{pg} / \mathrm{mL}$. Intra- and inter-assay variations of the assay were $<10 \%$ and $<12 \%$, respectively. Chymotrypsin concentration was measured using the Cusabio ELISA kit (Wuhan, Hubei Province, Chi$\mathrm{na}$ ), and results were expressed in $\mathrm{ng} / \mathrm{mL}$. Intra- and inter-assay variations of the assay were $<8 \%$ and $<10 \%$, respectively. Absorbance was detected at $450 \mathrm{~nm}$. The concentration of the enzymes in each sample was estimated using a standard curve.

\section{Covariates}

Fasting plasma glucose and fasting insulin were analysed using the above blood samples at LabPlus - a tertiary referral medical laboratory at Auckland City Hospital (Auckland, New Zealand). Fasting plasma glucose was measured using the enzymatic colorimetric assay (F.Hoffmann-La Roche Ltd., Basel, Switzerland). Fasting insulin was measured using the chemiluminescence sandwich immunoassay (Roche Diagnostics, Auckland, New Zealand). The weight and height of all participants were measured at the time of MR imaging to calculate body mass index.

\section{Statistical Analysis}

All statistical analyses were conducted using SAS version 9.4 for Windows (SAS Institute, Cary, NC, USA). Data were presented as median and interquartile range or frequency. The independent $t$-test and $\chi^{2}$ test were used to determine the differences in baseline characteristics between post-pancreatitis individuals and healthy controls. Linear regression analysis was conducted separately in 
Table 1. Characteristics of study participants

\begin{tabular}{|c|c|c|c|}
\hline Characteristic & Post-pancreatitis $(n=108)$ & Health $(n=60)$ & $p$ value \\
\hline Age, years & $58.0(44.0-66.0)$ & $49.0(30.5-67.5)$ & 0.035 \\
\hline Men, $n(\%)$ & $78(72.2)$ & $27(45.0)$ & 0.007 \\
\hline Body mass index, $\mathrm{kg} / \mathrm{m}^{2}$ & $27.8(24.5-32.7)$ & $24.2(21.5-27.6)$ & $<0.001$ \\
\hline Fasting plasma glucose, $\mathrm{mmol} / \mathrm{L}$ & $5.5(5.0-6.4)$ & $4.8(4.4-5.4)$ & $<0.001$ \\
\hline Fasting insulin, mlU/L & $10.4(6.3-16.5)$ & $9.9(5.4-13.4)$ & 0.064 \\
\hline \multicolumn{4}{|l|}{ Pancreatic enzymes } \\
\hline Amylase, U/L & $24.6(15.1-30.7)$ & $28.7(20.0-33.1)$ & 0.227 \\
\hline Lipase, pg/mL & $7.8(6.1-22.3)$ & $8.9(6.6-18.6)$ & 0.683 \\
\hline Chymotrypsin, ng/mL & $5.2(3.2-6.6)$ & $6.2(5.4-7.1)$ & 0.015 \\
\hline IPFD, \% & $9.5(8.6-10.4)$ & $7.7(6.4-9.2)$ & $<0.001$ \\
\hline $\mathrm{TPV}, \mathrm{cm}^{3}$ & $76.9(60.7-96.0)$ & $77.9(57.8-92.7)$ & 0.389 \\
\hline PHD, mm & $27.2(23.2-32.7)$ & $25.6(21.9-27.7)$ & 0.003 \\
\hline PBD, $\mathrm{mm}$ & $19.5(15.9-24.5)$ & $18.1(15.4-22.0)$ & 0.362 \\
\hline PTD, mm & $19.2(15.6-23.0)$ & $20.9(16.3-24.5)$ & 0.244 \\
\hline
\end{tabular}

Data are presented as median and interquartile range or percentage. IPFD, intra-pancreatic fat deposition; PBD pancreas body diameter; PHD, pancreas head diameter; PTD, pancreas tail diameter; TPV, total pancreas volume. $p$ values were from the independent $t$-test. Statistically significant values $(p<0.05)$ are in bold.

Post-pancreatitis individuals

\begin{tabular}{|c|c|c|c|c|c|c|c|c|c|c|c|c|c|c|c|c|}
\hline \multirow{4}{*}{ TPV } & Model & PHD & PBD & PTD & \multirow{4}{*}{ PHD } & PBD & PTD & TPV & \multirow{4}{*}{ PBD } & PTD & TPV & PHD & \multirow{4}{*}{ PTD } & TPV & PHD & PBD \\
\hline & 1 & 0.22 & 0.29 & 0.37 & & 0.22 & 0.08 & 0.03 & & 0.08 & 0.29 & 0.15 & & 0.37 & 0.03 & 0.15 \\
\hline & 2 & 0.28 & 0.35 & 0.42 & & 0.15 & 0.12 & 0.25 & & 0.18 & 0.29 & 0.12 & & 0.42 & 0.16 & 0.25 \\
\hline & 3 & 0.30 & 0.36 & 0.44 & & 0.20 & 0.18 & 0.50 & & 0.21 & 0.04 & 0.14 & & 0.43 & 0.19 & 0.27 \\
\hline
\end{tabular}

Healthy controls

\begin{tabular}{|c|c|c|c|c|c|c|c|c|c|c|c|c|c|c|c|c|}
\hline \multirow{4}{*}{ TPV } & Model & PHD & PBD & PTD & \multirow{4}{*}{ PHD } & PBD & PTD & TPV & \multirow{4}{*}{ PBD } & PTD & TPV & PHD & \multirow{4}{*}{ PTD } & TPV & PHD & PBD \\
\hline & 1 & 0.30 & 0.03 & 0.08 & & 0.30 & $<0.001$ & 0.03 & & $<0.001$ & 0.03 & $<0.001$ & & 0.08 & 0.03 & $<0.001$ \\
\hline & 2 & 0.47 & 0.28 & 0.33 & & 0.18 & 0.21 & 0.41 & & 0.01 & 0.04 & 0.01 & & 0.11 & 0.10 & 0.04 \\
\hline & 3 & 0.52 & 0.35 & 0.41 & & 0.32 & 0.36 & 0.29 & & 0.04 & 0.30 & 0.04 & & 0.12 & 0.13 & 0.04 \\
\hline
\end{tabular}

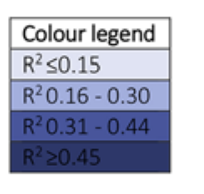

Fig. 2. Inter-relationships between TPV and the 3 diameters in post-pancreatitis individuals and healthy controls. Model 1, unadjusted; model 2, adjusted for age and sex; model 3, adjusted for age, sex, body mass index, and fasting glucose. Variables in rows are dependent variables, and variables in columns are independent variables. Colour coding is based on $R^{2}$ values and is applied to the models that were statistically significant. The models that were statistically insignificant have no colour coding. PBD, pancreas body diameter; PHD, pancreas head diameter; PTD, pancreas tail diameter; TPV, total pancreas volume.

post-pancreatitis individuals and healthy controls. The statistical analyses were conducted in 3 stages. First, to investigate the interrelationship between TPV and pancreas diameters (i.e., PHD, PBD, and PTD), each pancreas size variable (TPV, PHD, PBD, and PTD) was treated as both independent and dependent variables. Second, to investigate the associations between pancreas size (i.e., TPV, PHD, PBD, and PTD) and IPFD and pancreatic enzymes (i.e., pancreatic amylase, pancreatic lipase, and chymotrypsin), IPFD and pancreatic enzymes were treated as independent variables. Last, to investigate the associations between IPFD and pan- creatic enzymes (i.e., pancreatic amylase, pancreatic lipase, and chymotrypsin), IPFD was treated as an independent variable, and pancreatic enzymes were treated as dependent variables. The following 3 models were constructed for all the aforementioned analyses: (1) unadjusted, (2) adjusted for age and sex, (3) adjusted for age, sex, body mass index, and fasting plasma glucose. All data were reported as $\beta$ coefficients with corresponding standard errors and $p$ values. $p$ values $<0.05$ were deemed statistically significant. The most robust $R^{2}$ metric was yielded for each unadjusted model. 
Table 2. Inter-relationship between the studied pancreas size variables

\begin{tabular}{|c|c|c|c|c|c|c|c|c|}
\hline & \multicolumn{4}{|c|}{ Post-pancreatitis ( $n=108)$} & \multicolumn{4}{|c|}{ Health $(n=60)$} \\
\hline & B & SE & $p$ value & $R^{2}$ & B & SE & $p$ value & $R^{2}$ \\
\hline \multicolumn{9}{|l|}{ TPV } \\
\hline \multicolumn{9}{|l|}{ Model 1} \\
\hline PHD & 2.037 & 0.372 & $<0.001$ & 0.216 & 2.408 & 0.497 & $<0.001$ & 0.299 \\
\hline PBD & 2.410 & 0.368 & $<0.001$ & 0.285 & 0.753 & 0.553 & 0.179 & 0.033 \\
\hline PTD & 2.833 & 0.355 & $<0.001$ & 0.373 & 1.044 & 0.479 & 0.034 & 0.081 \\
\hline \multicolumn{9}{|l|}{ Model 2} \\
\hline PHD & 1.753 & 0.378 & $<0.001$ & & 2.217 & 0.485 & $<0.001$ & \\
\hline PBD & 2.103 & 0.368 & $<0.001$ & & 0.540 & 0.489 & 0.274 & \\
\hline PTD & 2.641 & 0.374 & $<0.001$ & & 0.860 & 0.427 & 0.049 & \\
\hline \multicolumn{9}{|l|}{ Model 3} \\
\hline PHD & 1.668 & 0.394 & $<0.001$ & & 2.114 & 0.545 & $<0.001$ & \\
\hline PBD & 1.980 & 0.376 & $<0.001$ & & 0.317 & 0.533 & 0.555 & \\
\hline PTD & 2.523 & 0.379 & $<0.001$ & & 0.816 & 0.416 & 0.057 & \\
\hline \multicolumn{9}{|l|}{ PHD } \\
\hline \multicolumn{9}{|l|}{ Model 1} \\
\hline TPV & 0.106 & 0.019 & $<0.001$ & 0.216 & 0.124 & 0.026 & $<0.001$ & 0.299 \\
\hline PBD & 0.301 & 0.097 & 0.003 & 0.082 & 0.049 & 0.127 & 0.705 & 0.003 \\
\hline PTD & 0.205 & 0.105 & 0.055 & 0.034 & 0.149 & 0.116 & 0.205 & 0.030 \\
\hline \multicolumn{9}{|l|}{ Model 2} \\
\hline TPV & 0.095 & 0.021 & $<0.001$ & & 0.128 & 0.028 & $<0.001$ & \\
\hline PBD & 0.231 & 0.098 & 0.020 & & 0.008 & 0.119 & 0.948 & \\
\hline PTD & 0.142 & 0.110 & 0.201 & & 0.174 & 0.089 & 0.122 & \\
\hline \multicolumn{9}{|l|}{ Model 3} \\
\hline TPV & 0.088 & 0.021 & $<0.001$ & & 0.127 & 0.033 & $<0.001$ & \\
\hline PBD & 0.185 & 0.098 & 0.063 & & 0.052 & 0.131 & 0.693 & \\
\hline PTD & 0.106 & 0.110 & 0.337 & & 0.219 & 0.109 & 0.053 & \\
\hline \multicolumn{9}{|l|}{ PBD } \\
\hline \multicolumn{9}{|l|}{ Model 1} \\
\hline TPV & 0.118 & 0.018 & $<0.001$ & 0.285 & 0.043 & 0.032 & 0.179 & 0.033 \\
\hline PHD & 0.271 & 0.087 & 0.003 & 0.082 & 0.054 & 0.142 & 0.705 & 0.003 \\
\hline PTD & 0.401 & 0.093 & $<0.001$ & 0.147 & 0.007 & 0.125 & 0.959 & $<0.001$ \\
\hline \multicolumn{9}{|l|}{ Model 2} \\
\hline TPV & 0.112 & 0.020 & $<0.001$ & & 0.042 & 0.038 & 0.274 & \\
\hline PHD & 0.215 & 0.091 & 0.020 & & 0.010 & 0.159 & 0.948 & \\
\hline PTD & 0.372 & 0.100 & $<0.001$ & & 0.003 & 0.129 & 0.984 & \\
\hline \multicolumn{9}{|l|}{ Model 3} \\
\hline TPV & 0.106 & 0.020 & $<0.001$ & & 0.027 & 0.045 & 0.555 & \\
\hline PHD & 0.178 & 0.095 & 0.063 & & 0.074 & 0.186 & 0.693 & \\
\hline PTD & 0.339 & 0.102 & 0.001 & & 0.028 & 0.135 & 0.835 & \\
\hline \multicolumn{9}{|l|}{ PTD } \\
\hline \multicolumn{9}{|l|}{ Model 1} \\
\hline TPV & 0.132 & 0.017 & $<0.001$ & 0.373 & 0.077 & 0.036 & 0.034 & 0.081 \\
\hline PHD & 0.166 & 0.086 & 0.055 & 0.034 & 0.199 & 0.155 & 0.205 & 0.030 \\
\hline PBD & 0.367 & 0.085 & $<0.001$ & 0.147 & 0.008 & 0.148 & 0.959 & $<0.001$ \\
\hline
\end{tabular}


Table 2 (continued)

\begin{tabular}{|c|c|c|c|c|c|c|c|c|}
\hline & \multicolumn{4}{|c|}{ Post-pancreatitis $(n=108)$} & \multicolumn{4}{|c|}{ Health $(n=60)$} \\
\hline & B & SE & $p$ value & $R^{2}$ & B & SE & $p$ value & $R^{2}$ \\
\hline \multicolumn{9}{|l|}{ Model 2} \\
\hline TPV & 0.122 & 0.017 & $<0.001$ & & 0.084 & 0.042 & 0.049 & \\
\hline PHD & 0.109 & 0.085 & 0.201 & & 0.287 & 0.167 & 0.091 & \\
\hline PBD & 0.312 & 0.084 & $<0.001$ & & 0.003 & 0.149 & 0.984 & \\
\hline \multicolumn{9}{|l|}{ Model 3} \\
\hline TPV & 0.119 & 0.018 & $<0.001$ & & 0.107 & 0.055 & 0.057 & \\
\hline PHD & 0.085 & 0.088 & 0.337 & & 0.414 & 0.208 & 0.053 & \\
\hline PBD & 0.287 & 0.086 & 0.001 & & 0.039 & 0.185 & 0.835 & \\
\hline
\end{tabular}

Data are presented as $\beta$ coefficients, standard errors, $p$ values (from linear regression), and $R^{2}$ values (from the unadjusted model). Model 1: unadjusted model. Model 2: adjusted for age and sex. Model 3: adjusted for age, sex, body mass index, and fasting plasma glucose. PBD, pancreas body diameter; PHD, pancreas head diameter; PTD, pancreas tail diameter; TPV, total pancreas volume. $p$ values were from linear regression analyses. Statistically significant values $(p<0.05)$ are in bold.

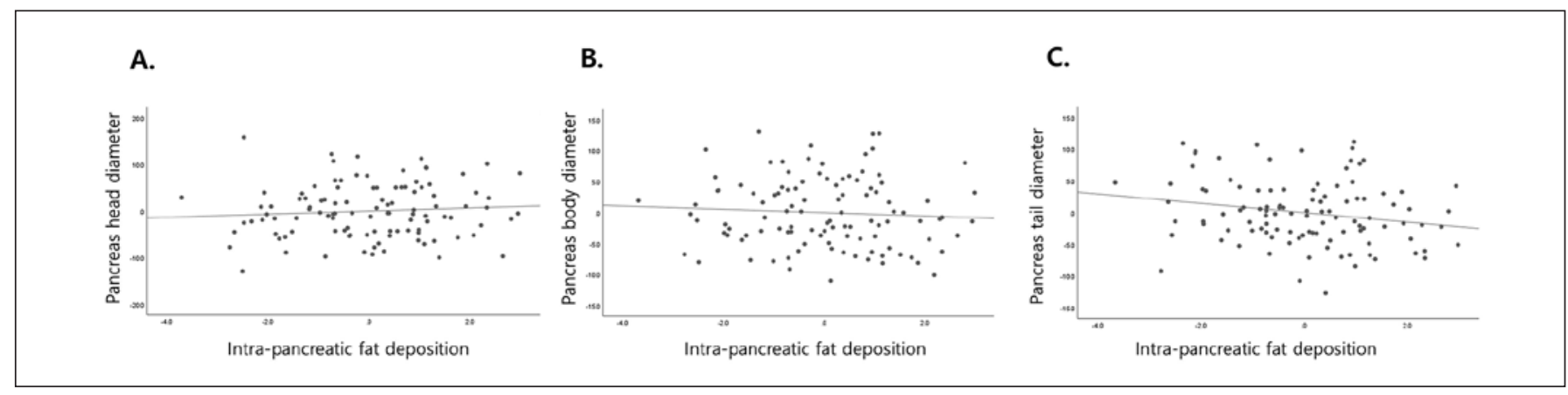

Fig. 3. Associations between IPFD and $\operatorname{PHD}(\mathbf{A}), \operatorname{PBD}(\mathbf{B})$, and PTD (C) in post-pancreatitis individuals. All partial residual plots were adjusted for age, sex, body mass index, and fasting plasma glucose. IPFD, intra-pancreatic fat deposition; PBD, pancreas body diameter; PHD, pancreas head diameter; PTD, pancreas tail diameter.

\section{Results}

\section{Characteristics of the Study Groups}

A total of 168 individuals were included, of whom 108 were post-pancreatitis individuals and 60 were healthy controls. Post-pancreatitis individuals were studied at 20 months (interquartile range, 12-29 months) since their last attack of AP. Detailed characteristics of the study groups are presented in Table 1.

\section{Pancreas Size in the Study Groups}

The median TPV $\left(\mathrm{cm}^{3}\right)$ was $76.9 \mathrm{~cm}^{3}$ (interquartile range, $60.7-96.0 \mathrm{~cm}^{3}$ ) in the post-pancreatitis group and $77.9 \mathrm{~cm}^{3}$ (interquartile range, $57.8-92.7 \mathrm{~cm}^{3}$ ) in the control group ( $p=0.389)$. The ICC of TPV measurements was 0.97 .
In the post-pancreatitis group, TPV was significantly associated with PHD in both the unadjusted $(\beta=2.037, p$ $<0.001)$ and all the adjusted models $(p<0.001$ in model $2 ; p<0.001$ in model 3) (Fig. 2). TPV was significantly associated with PBD in both the unadjusted $(\beta=2.410, p<$ $0.001)$ and all the adjusted models $(p<0.001$ in model 2; $p<0.001$ in model 3 ). TPV was significantly associated with PTD in both the unadjusted $(\beta=2.833, p<0.001)$ and all the adjusted models $(p<0.001$ in model 2 ; $p<$ 0.001 in model 3). Inter-relationships between PHD and PBD as well as between PHD and PTD were not significant in the adjusted models (Table 2).

In the control group, TPV was significantly associated with PHD in both the unadjusted $(\beta=2.408, p<0.001)$ and all the adjusted models $(p<0.001$ in model 2; $p<$ 
Table 3. Associations of pancreas size with IPFD and pancreatic enzymes

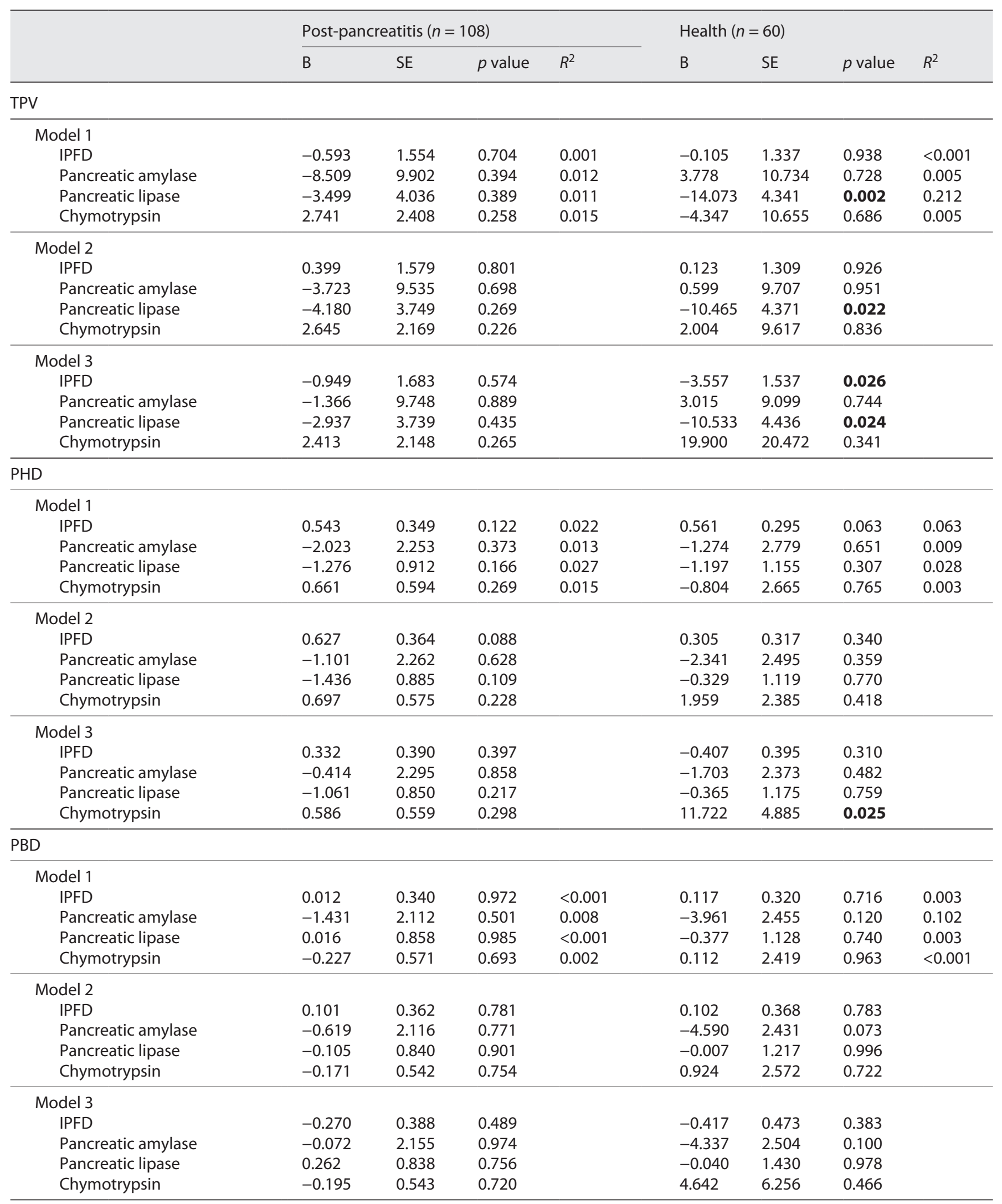


Table 3 (continued)

\begin{tabular}{|c|c|c|c|c|c|c|c|c|}
\hline & \multicolumn{4}{|c|}{ Post-pancreatitis $(n=108)$} & \multicolumn{4}{|c|}{ Health $(n=60)$} \\
\hline \multicolumn{9}{|l|}{ Model 1} \\
\hline IPFD & -0.725 & 0.310 & 0.021 & 0.049 & -0.627 & 0.337 & 0.069 & 0.061 \\
\hline Pancreatic amylase & 2.378 & 2.024 & 0.245 & 0.023 & -1.140 & 3.035 & 0.711 & 0.006 \\
\hline Chymotrypsin & 0.482 & 0.573 & 0.402 & 0.009 & 0.982 & 2.832 & 0.731 & 0.004 \\
\hline \multicolumn{9}{|l|}{ Model 2} \\
\hline IPFD & -0.423 & 0.323 & 0.194 & & -0.511 & 0.384 & 0.189 & \\
\hline Pancreatic amylase & 3.572 & 1.840 & 0.057 & & -1.395 & 2.804 & 0.624 & \\
\hline Pancreatic lipase & 0.105 & 0.778 & 0.894 & & -0.570 & 1.255 & 0.653 & \\
\hline Chymotrypsin & 0.585 & 0.505 & 0.250 & & 0.749 & 3.030 & 0.807 & \\
\hline Pancreatic lipase & 0.365 & 0.786 & 0.644 & & -0.941 & 1.408 & 0.510 & \\
\hline Chymotrypsin & 0.539 & 0.506 & 0.290 & & -2.313 & 7.333 & 0.756 & \\
\hline
\end{tabular}

Data are presented as $\beta$ coefficients, standard errors, $p$ values (from linear regression), and $R^{2}$ values (from the unadjusted analysis). Pancreatic amylase, pancreatic lipase, and chymotrypsin data were log-transformed. Model 1: unadjusted model. Model 2: adjusted for age and sex. Model 3: adjusted for age, sex, body mass index, and fasting plasma glucose. IPFD, intra-pancreatic fat deposition; PBD, pancreas body diameter; PHD, pancreas head diameter; PTD, pancreas tail diameter; TPV, total pancreas volume. $p$ values were from linear regression analyses. Statistically significant values $(p<0.05)$ are in bold.

0.001 in model 3) (Fig. 2). TPV was significantly associated with PTD in the unadjusted $(\beta=1.044, p=0.034)$ model, but not in the most adjusted model $(p=0.057$ in model 3). Inter-relationships between 3 pancreas diameters were not significant in both unadjusted and adjusted models (Table 2).

Associations between Pancreas Size and IPFD in the Study Groups

The median IPFD (\%) was 9.5\% (interquartile range, $8.6-10.4 \%$ ) in the post-pancreatitis group and $7.7 \%$ (interquartile range, $6.4-9.2 \%)$ in the control group $(p<$ 0.001). The ICC of IPFD measurements was 0.97 .

In the post-pancreatitis group, IPFD was significantly associated with PTD in both the unadjusted $(\beta=-0.725$, $p=0.021)$ and the most adjusted model $(p=0.036$ in model 3) (Fig. 3). Other pancreas size variables were not significantly associated with IPFD in any model (Table 3$)$.

In the control group, IPFD was significantly associated with TPV in the most adjusted model ( $p=0.026$ in model 3). The other pancreas size variables were not significantly associated with IPFD in any model (Table 3 ).

\section{Associations between Pancreas Size and Pancreatic} Enzymes in the Study Groups

In the post-pancreatitis group, pancreatic amylase was significantly associated with PTD in the most adjusted model ( $p=0.042$ in model 3) (Fig. 4). Other pancreas size variables were not significantly associated with pancreatic enzymes in any model (Table 3 ).

In the control group, pancreatic lipase was significantly associated with TPV in both the unadjusted $(\beta=$ $-14.073, p=0.002)$ and all the adjusted models $(p=0.022$ in model 2; $p=0.024$ in model 3). Chymotrypsin was significantly associated with PHD in the most adjusted model $(p=0.025$ in model 3$)$. The other pancreas size variables were not significantly associated with pancreatic enzymes in any model (Table 3).

\section{Associations between IPFD and Pancreatic Enzymes in the Study Groups}

In both the post-pancreatitis group and the control group, there were no statistically significant associations between IPFD and the studied pancreatic enzymes in all models (Table 4). 
Table 4. Associations of IPFD with pancreatic enzymes

\begin{tabular}{|c|c|c|c|c|c|c|c|c|}
\hline & \multicolumn{4}{|c|}{ Post-pancreatitis $(n=108)$} & \multicolumn{4}{|c|}{ Health $(n=60)$} \\
\hline & B & SE & $p$ value & $R^{2}$ & B & SE & $p$ value & $R^{2}$ \\
\hline \multicolumn{9}{|l|}{ IPFD } \\
\hline \multicolumn{9}{|l|}{ Model 1} \\
\hline Pancreatic amylase & -0.011 & 0.024 & 0.640 & 0.003 & -0.045 & 0.041 & 0.278 & 0.049 \\
\hline Pancreatic lipase & -0.049 & 0.055 & 0.373 & 0.011 & 0.028 & 0.064 & 0.664 & 0.005 \\
\hline Chymotrypsin & 0.047 & 0.070 & 0.505 & 0.005 & -0.031 & 0.031 & 0.315 & 0.032 \\
\hline \multicolumn{9}{|l|}{ Model 2} \\
\hline Pancreatic amylase & -0.013 & 0.026 & 0.621 & & -0.047 & 0.043 & 0.287 & \\
\hline Pancreatic lipase & -0.069 & 0.058 & 0.242 & & 0.046 & 0.064 & 0.473 & \\
\hline Chymotrypsin & 0.032 & 0.076 & 0.676 & & -0.024 & 0.032 & 0.457 & \\
\hline \multicolumn{9}{|l|}{ Model 3} \\
\hline Pancreatic amylase & 0.003 & 0.027 & 0.900 & & -0.067 & 0.059 & 0.273 & \\
\hline Pancreatic lipase & -0.044 & 0.060 & 0.464 & & 0.090 & 0.087 & 0.307 & \\
\hline Chymotrypsin & 0.033 & 0.082 & 0.686 & & 0.010 & 0.021 & 0.628 & \\
\hline
\end{tabular}

Data are presented as $\beta$ coefficients, standard errors, $p$ values (from linear regression), and $R^{2}$ values (from the unadjusted analysis). Pancreatic amylase, pancreatic lipase, and chymotrypsin data were log-transformed. Model 1: unadjusted model. Model 2: adjusted for age and sex. Model 3: adjusted for age, sex, body mass index, and fasting plasma glucose. IPFD, intra-pancreatic fat deposition. $p$ values were from linear regression analyses. Statistically significant values $(p<0.05)$ are in bold.

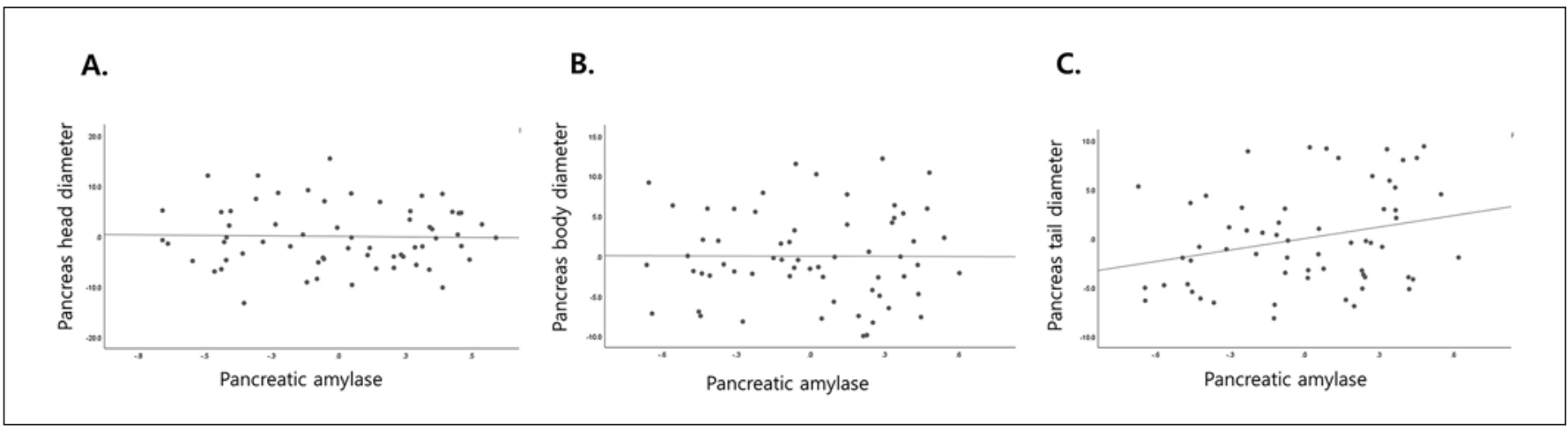

Fig. 4. Associations between pancreatic amylase and $\mathrm{PHD}(\mathbf{A}), \operatorname{PBD}(\mathbf{B})$, and PTD $(\mathbf{C})$ in post-pancreatitis individuals. All partial residual plots were adjusted for age, sex, body mass index, and fasting plasma glucose. PBD, pancreas body diameter; PHD, pancreas head diameter; PTD, pancreas tail diameter.

\section{Discussion}

The present study comprehensively investigated the associations between pancreas morphology derived from 3T MR imaging and pancreatic enzymes in post-pancreatitis individuals. It presented the first data on differential associations between IPFD and pancreas size (including TPV and diameters of the head, body, and tail of the pancreas) in the post-pancreatitis setting. Further, this study provided evidence on associations between pancreas morphology and 3 pancreatic enzymes - pancreatic amylase, pancreatic lipase, and chymotrypsin (Fig. 5). All MR imaging-derived measurements, performed by 2 independent raters, had excellent inter-rater reliability (ICC of 0.97 for both IPFD and TPV). The main finding of this study was that increased IPFD was significantly associated with reduced PTD (but not TPV, PHD, or PBD) in the post-pancreatitis group. Also, increased IPFD was 
A.

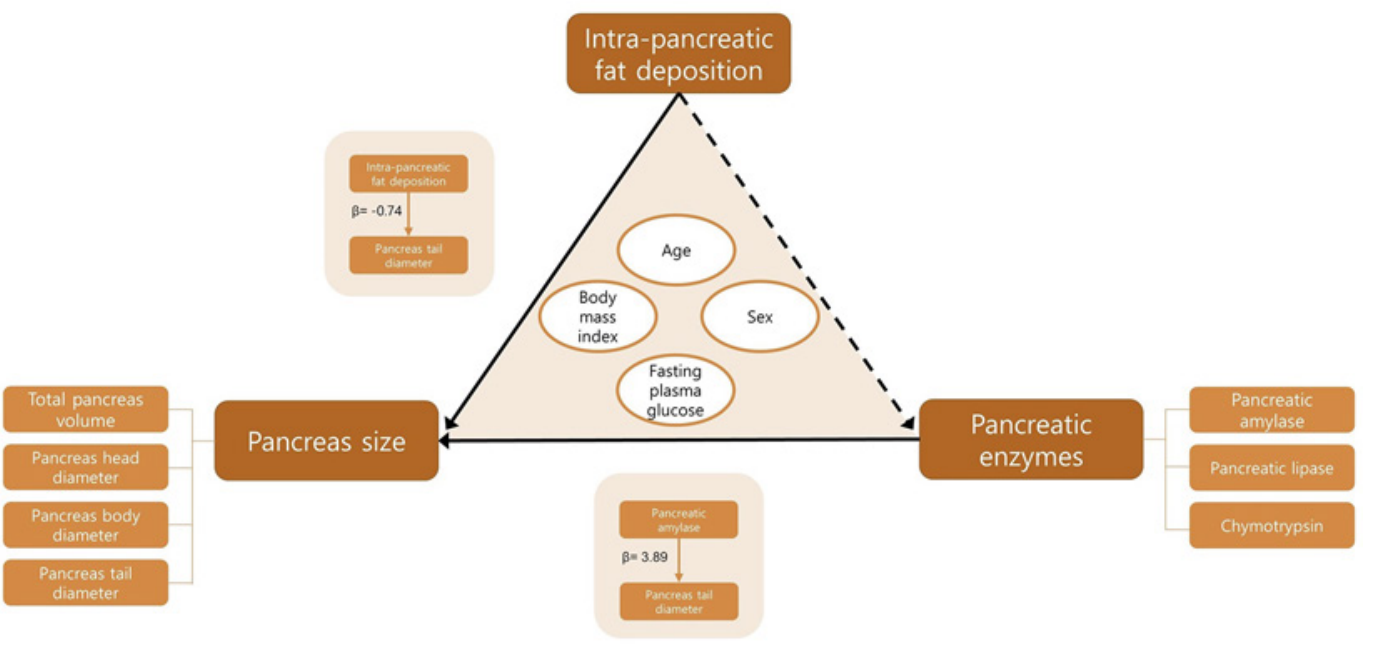

B.

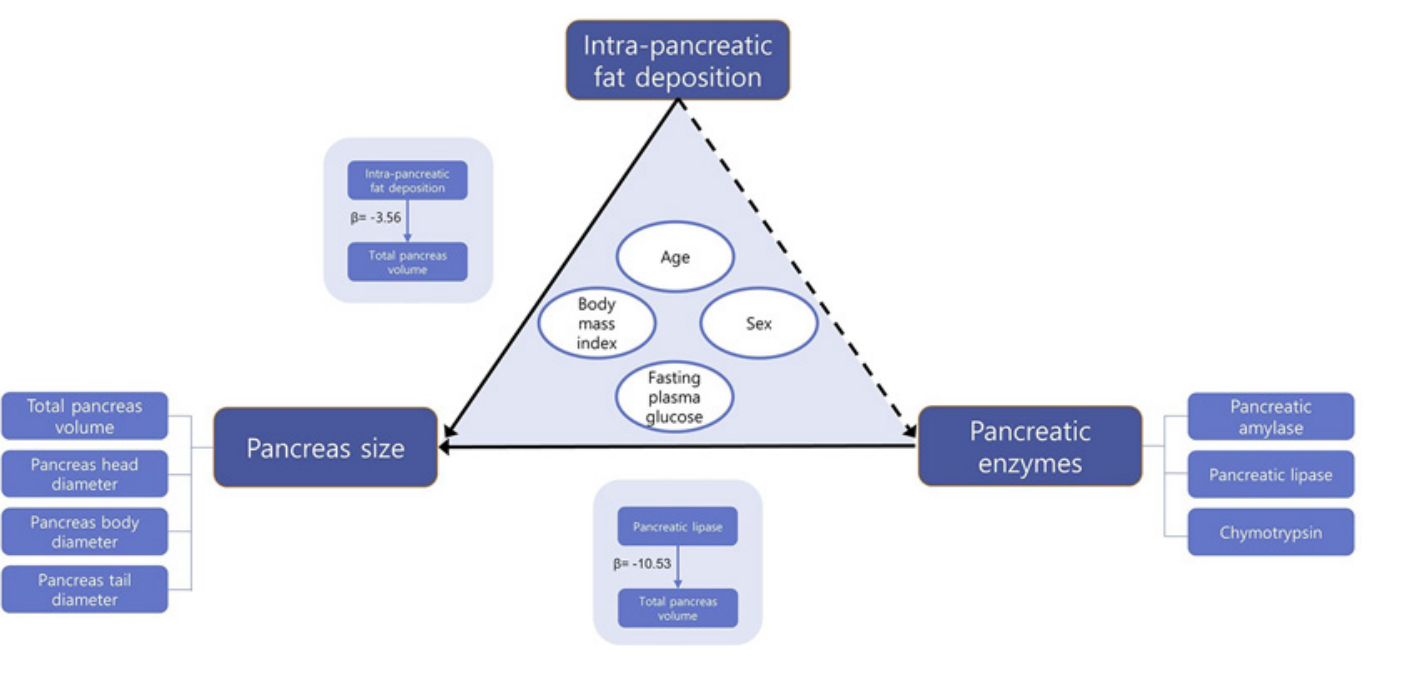

Fig. 5. Summary of the data analysis and key findings in post-pancreatitis individuals (A) and healthy individuals (B). Single-headed arrows indicate regression paths. Solid lines indicate statistically significant paths, and dashed lines indicate not-significant paths. $\beta$-Coefficients are shown for specific statistically significant asso- ciations. Ovals represent the covariates that were adjusted for. IPFD, intra-pancreatic fat deposition; PBD, pancreas body diameter; PHD, pancreas head diameter; PTD, pancreas tail diameter; $\mathrm{TPV}$, total pancreas volume. significantly associated with reduced TPV (but not PHD, $\mathrm{PBD}$, or PTD) in the control group. Further, of the 3 pancreatic enzymes investigated, pancreatic amylase was directly associated with PTD in the post-pancreatitis group, whereas pancreatic lipase was inversely associated with TPV in the control group. Notably, the above findings were independent of age, sex, body mass index, and fasting plasma glucose - variables found to considerably affect pancreas size in the systematic reviews by the COSMOS group conducted to inform the design of the ARIES project $[8,10,15]$. 
Consistent evidence shows that TPV is inversely associated with IPFD and decreases further with the time since onset of metabolic disorders. A longitudinal study from the UK found that an increase in TPV was associated with the remission of type 2 diabetes following dietary weight loss during a 24-month follow-up [21]. The authors suggested that a decrease in IPFD and restoration of $\beta$-cell function were associated with gradual normalization of the pancreas size [21]. A longitudinal MR study in which individuals with chronic pancreatitis were followed up for 4 years showed a significantly reduced TPV and increased IPFD in comparison with baseline measurements [22]. In addition to TPV reduction, a reduction in the size of the tail of the pancreas in individuals with metabolic disorders was reported. Specifically, in the setting of type 1 diabetes, the tail of the pancreas was the first to shrink and displayed the greatest extent of atrophy relative to the head and body regions of the pancreas [15]. Similarly, a 2019 MR study found pancreas tail shrinkage in individuals with recurrent attacks of AP [8]. In the present study, each unit increase in IPFD was significantly associated with a $0.7-\mathrm{mm}$ reduction in PTD; and a 10$\mathrm{U} / \mathrm{L}$ reduction in circulating levels of pancreatic amylase was significantly associated with a $0.4-\mathrm{mm}$ reduction in PTD in the post-pancreatitis group (independent of age, sex, body mass index, and fasting plasma glucose). The finding of a higher IPFD in the post-pancreatitis group than in the control group (9.5\% [interquartile range: 8.610.4 ] versus $7.7 \%$ [interquartile range: $6.4-9.2$ ]; $p<0.001$ ) and the significant inverse association between IPFD and PTD suggest that IPFD might be involved in the pathogenesis of exocrine pancreatic dysfunction $[5,23]$. However, in the present study, while PTD was significantly associated with both IPFD and pancreatic amylase, there was no association between IPFD and pancreatic amylase (or the other pancreatic enzymes) in the post-pancreatitis group. The mechanisms underlying these inter-relations remain unclear, and the effect of a residual confounder cannot be ruled out [24]. Prospective cohort studies are now warranted to investigate whether the abnormal pancreas morphology can be reversed with the use of interventions (e.g., diet modifications) in individuals after clinical resolution of AP $[25,26]$.

Another notable finding in the present study relates to healthy controls. In the present study, each unit reduction in IPFD was significantly associated with a $3.6-\mathrm{cm}^{3}$ increase in TPV; and a $10 \mathrm{pg} / \mathrm{mL}$ reduction in circulating levels of pancreatic lipase was significantly associated with a $1.1-\mathrm{cm}^{3}$ increase in TPV (independent of age, sex, body mass index, and fasting plasma glucose). This find- ing is broadly in line with previous studies that showed a clinical relevance of IPFD to exocrine pancreatic function. An earlier population-based study found that MR imaging-derived IPFD was inversely associated with circulating levels of serum lipase (unlike the present study, pancreatic amylase and chymotrypsin were not investigated) in the general population, suggesting that increased IPFD was associated with reduced acinar cell mass of the pancreas [27]. Moreover, a 2019 study of 1,458 individuals showed that MR imaging-derived IPFD was inversely associated with exocrine pancreatic function (as measured by fecal elastase), in both crude analysis and after adjustment for age, sex, and body mass index [28]. Another study of MR imaging-derived IPFD investigated individuals without a history of diabetes or pancreatitis and demonstrated a significant exocrine pancreatic dysfunction (as measured by fecal elastase) in individuals with excess IPFD compared with controls [29]. Taken together, increased IPFD in generally healthy people could be a harbinger of exocrine pancreatic dysfunction and possible subsequent metabolic diseases [30-32].

There are further points to consider when interpreting the results of the present study. First, this was a crosssectional study with an average of nearly 2 years after an attack of pancreatitis. Longitudinal studies are warranted to capture temporal changes in pancreas morphology with a view to drawing causal inferences. Second, levels of amylase, lipase, and chymotrypsin in the gastrointestinal tract were not measured. Hence, it is unknown whether their circulating and intraluminal levels were associated in the study groups. However, a 2020 systematic review and meta-analysis by the COSMOS group identified circulating levels of serum amylase and lipase as biomarkers of several metabolic disorders [14]. Third, histological data were not available due to the technical difficulties associated with pancreas biopsy. Further, such a procedure would be unethical in our study population. Last, the reported findings cannot be extrapolated to the pediatric setting as pancreas size typically increases from infancy to adolescence [10, 15].

\section{Conclusion}

This study holistically investigated morphological changes in the pancreas (determined with the use of 3T MR imaging for each study participant by 2 independent raters in a blinded fashion), encompassing IPFD, TPV, and regional pancreas diameters in both individuals after AP and healthy individuals. Our results showed that in-
Obes Facts 2022;15:70-82 DOI: $10.1159 / 000519621$
Ko/Skudder-Hill/Priya/Kimita/Bharmal/ Petrov 
creased IPFD was significantly associated with reduced PTD in individuals after AP and reduced TPV in healthy individuals (Fig. 5). Further, PTD was directly associated with pancreatic amylase in individuals after AP, whereas TPV was inversely associated with pancreatic lipase in healthy individuals. These findings were irrespective of age, sex, body mass index, and fasting plasma glucose. Deposition of fat in the pancreas has implications for the exocrine pancreas in both health and disease.

\section{Acknowledgments}

This study was part of the COSMOS programme.

\section{Statement of Ethics}

The study was approved by the Health and Disability Ethics Committee (13/STH/182). All participants provided their written informed consent.

\section{Conflict of Interest Statement}

The authors have no conflict of interest.

\section{Funding Sources}

This study was supported by the Royal Society of New Zealand (Rutherfold Discovery Fellowship to Professor Max Petrov), which played no role in the study design, collection, analysis, interpretation of data, or writing of the manuscript.

\section{Author Contributions}

Conceptualization and the study design were conceived by M.S.P.; patient recruitment was conducted by J.K., S.P., and S.H.B.; data acquisition was performed by J.K., L.S.H., S.P., W.K., and S.H.B.; analysis and interpretation of data were performed by J.K.; statistical analysis was conducted by J.K.; drafting of the manuscript was performed by J.K.; revision of the manuscript was performed by L.S.H., S.P., W.K., S.H.B., and M.S.P.; study supervision was performed by M.S.P.

\section{Data Availability Statement}

All data generated or analysed during this study are included in the manuscript.

\section{References}

1 Xiao AY, Tan ML, Wu LM, Asrani VM, Windsor JA, Yadav D, et al. Global incidence and mortality of pancreatic diseases: a systematic review, meta-analysis, and meta-regression of population-based cohort studies. Lancet Gastroenterol Hepatol. 2016;1(1):4555.

2 Petrov MS, Yadav D. Global epidemiology and holistic prevention of pancreatitis. Nat Rev Gastroenterol Hepatol. 2019;16(3):17584.

3 Bharmal SH, Cho J, Alarcon Ramos GC, Ko J, Stuart CE, Modesto AE, et al. Trajectories of glycaemia following acute pancreatitis: a prospective longitudinal cohort study with 24 months follow-up. J Gastroenterol. 2020;55: $775-88$.

4 Das SL, Singh PP, Phillips AR, Murphy R, Windsor JA, Petrov MS. Newly diagnosed diabetes mellitus after acute pancreatitis: a systematic review and meta-analysis. Gut. 2014; 63(5):818-31.

5 Cho J, Scragg R, Pandol SJ, Petrov MS. Exocrine pancreatic dysfunction increases the risk of new-onset diabetes mellitus: results of a nationwide cohort study. Clin Transl Sci. 2021;14(1):170-8.
6 Ko J, Skudder-Hill L, Cho J, Bharmal SH, Petrov MS. The relationship between abdominal fat phenotypes and insulin resistance in non-obese individuals after acute pancreatitis. Nutrients. 2020;12(9):1-14.

7 Singh RG, Cervantes A, Kim JU, Nguyen NN, DeSouza SV, Dokpuang D, et al. Intrapancreatic fat deposition and visceral fat volume are associated with the presence of diabetes after acute pancreatitis. Am J Physiol Gastrointest Liver Physiol. 2019;316(6):G806-15.

8 DeSouza SV, Priya S, Cho J, Singh RG, Petrov MS. Pancreas shrinkage following recurrent acute pancreatitis: an MRI study. Eur Radiol. 2019;29(7):3746-56.

9 Burute N, Nisenbaum R, Jenkins DJ, Mirrahimi A, Anthwal S, Colak E, et al. Pancreas volume measurement in patients with type 2 diabetes using magnetic resonance imaging-based planimetry. Pancreatology. 2014;14(4):268-74.

10 DeSouza SV, Singh RG, Yoon HD, Murphy R, Plank LD, Petrov MS. Pancreas volume in health and disease: a systematic review and meta-analysis. Expert Rev Gastroenterol Hepatol. 2018;12(8):757-66.
11 Lim S, Bae JH, Chun EJ, Kim H, Kim SY, Kim $\mathrm{KM}$, et al. Differences in pancreatic volume, fat content, and fat density measured by multidetector-row computed tomography according to the duration of diabetes. Acta Diabetol. 2014;51(5):739-48.

12 Madzak A, Olesen SS, Haldorsen IS, Drewes AM, Frøkjær JB. Secretin-stimulated MRI characterization of pancreatic morphology and function in patients with chronic pancreatitis. Pancreatology. 2017;17(2):228-36.

13 Singh RG, Yoon HD, Wu LM, Lu J, Plank LD, Petrov MS. Ectopic fat accumulation in the pancreas and its clinical relevance: a systematic review, meta-analysis, and meta-regression. Metabolism. 2017;69(4):1-13.

14 Ko J, Cho J, Petrov MS. Low serum amylase, lipase, and trypsin as biomarkers of metabolic disorders: a systematic review and meta-analysis. Diabetes Res Clin Pract. 2020;159(1): 107974.

15 DeSouza SV, Yoon HD, Singh RG, Petrov MS Quantitative determination of pancreas size using anatomical landmarks and its clinical relevance: a systematic literature review. Clin Anat. 2018;31(6):913-26. 
16 Cervantes A, Singh RG, Kim JU, DeSouza SV, Petrov MS. Relationship of anthropometric indices to abdominal body composition: a multi-ethnic New Zealand magnetic resonance imaging study. J Clin Med Res. 2019; 11(6):435-46.

17 Singh RG, Nguyen NN, Cervantes A, Alarcon Ramos GC, Cho J, Petrov MS. Associations between intra-pancreatic fat deposition and circulating levels of cytokines. Cytokine. 2019;120(8):107-14.

18 Singh RG, Nguyen NN, DeSouza SV, Pendharkar SA, Petrov MS. Comprehensive analysis of body composition and insulin traits associated with intra-pancreatic fat deposition in healthy individuals and people with newonset prediabetes/diabetes after acute pancreatitis. Diabetes Obes Metab. 2019;21(2):41723.

19 Dellinger EP, Forsmark CE, Layer P, Lévy P, Maraví-Poma E, Petrov MS, et al. Determinant-based classification of acute pancreatitis severity: an international multidisciplinary consultation. Ann Surg. 2012;256(6):875-80.

20 Al-Mrabeh A, Hollingsworth KG, Steven S, Tiniakos D, Taylor R. Quantification of intrapancreatic fat in type 2 diabetes by MRI. PLoS One. 2017;12(4):e0174660-19.
21 Al-Mrabeh A, Hollingsworth KG, Shaw JA, Mcconnachie A, Sattar N, Lean ME, et al. 2 -year remission of type 2 diabetes and pancreas morphology : a post-hoc analysis of the DiRECT open-label, cluster-randomised trial. Lancet Diabetes Endocrinol. 2020;8(20): 939-48.

22 Steinkohl E, Olesen SS, Mark EB, Hansen TM, Frandsen LK, Drewes AM, et al. Progression of parenchymal and ductal findings in patients with chronic pancreatitis: a 4-year follow-up MRI study. Eur J Radiol. 2020;125(4): 108868.

23 Das SL, Kennedy JI, Murphy R, Phillips AR, Windsor JA, Petrov MS. Relationship between the exocrine and endocrine pancreas after acute pancreatitis. World J Gastroenterol. 2014;20(45):17196-205.

24 Ko J, Skudder-Hill L, Cho J, Bharmal SH, Petrov MS. Pancreatic enzymes and abdominal adipose tissue distribution in new-onset prediabetes/diabetes after acute pancreatitis. World J Gastroenterol. 2021;27(23):3357-71.

25 Ko J, Skudder-Hill L, Tarrant C, Kimita W, Bharmal SH, Petrov MS. Intra-pancreatic fat deposition as a modifier of the relationship between habitual dietary fat intake and insulin resistance. Clin Nutr. 2021;40(7):4730-7.

26 Ko J, Kimita W, Skudder-Hill L, Li X, Priya S, Bharmal SH, et al. Dietary carbohydrate intake and insulin traits in individuals after acute pancreatitis: effect modification by intra-pancreatic fat deposition. Pancreatology. 2021;21(2):353-62.
27 Kühn JP, Berthold F, Mayerle J, Völzke H, Reeder SB, Rathmann W, et al. Pancreatic steatosis demonstrated at MR imaging in the general population: clinical relevance. Radiology. 2015;276(1):129-36.

28 Kromrey ML, Friedrich N, Hoffmann RT, Bülow R, Völzke H, Weiss FU, et al. Pancreatic steatosis is associated with impaired exocrine pancreatic function. Invest Radiol. 2019; 54(7):403-8.

29 Tahtacı M, Algın O, Karakan T, Yürekli OT, Alışık M, Köseoğlu H, et al. Can pancreatic steatosis affect exocrine functions of pancreas? Turkish J Gastroenterol. 2018;29(5):58894.

30 Bharmal SH, Alarcon Ramos GC, Ko J, Petrov MS. Abdominal fat distribution modulates the metabolic effects of exogenous ketones in individuals with new-onset prediabetes after acute pancreatitis: results from a randomized placebo-controlled trial. Clin Nutr ESPEN. 2021;43:117-29.

31 Petrov MS. Post-pancreatitis diabetes mellitus: investigational drugs in preclinical and clinical development and therapeutic implications. Expert Opin Investig Drugs. 2021; 30(7):737-47.

32 Petrov MS. Post-pancreatitis diabetes mellitus: prime time for secondary disease. Eur J Endocrinol. 2021;184(4):R137-49. 